\title{
Estudio comparativo in vitro de residuos extruidos apicalmente utilizando Sistemas de Lima Única con y sin ensanchamiento previo del conducto radicular
}

Comparative in vitro study of apical extruded waste using Single-File Systems with and without prior root canal widening

Recibido: 2015/12/15. Aceptado: 2016/08/02. Publicado: 2018/03/01

\section{Daniel Izquierdo Camacho ${ }^{1}$ Andrea Ponce Bueno ${ }^{2}$}

1 Universidad San Francisco de Quito, Colegio de Ciencias de la Salud, Escuela de Odontología, Clínica Odontológica, Campus Cumbayá, oficina CO 106, casilla postal 17-1200-841. Quito.

Correo electrónico: dr.dsic@gmail.com

2 Universidad San Francisco de Quito, Colegio de Ciencias de la Salud, Escuela de Odontología, Clínica Odontológica, Campus Cumbayá, oficina CO 106, casilla postal 17-1200-841. Quito.

Correo electrónico: andreaponce17@gmail.com 


\section{Resumen}

El propósito de este estudio in vitro fue comparar la cantidad de detritos dentinarios extruidos en miligramos al periápice después de la instrumentación rotatoria con dos tipos de instrumentos reciprocantes, incluyendo o no un ensanchamiento previo del conducto radicular o Glide path. Cuarenta raíces mesiales de molares inferiores se instrumentaron a la longitud de trabajo establecida, divididas en cuatro grupos (G) de estudio. En el G1 se utilizó PathFile 1 - 2 - 3 (Dentsply Maillefer) y luego Reciproc R25 0,08 (VDW GmbH, Munich Germany). En el G2 se instrumentó con Reciproc R25. En el G3 se utilizó PathFile 1 - 2 -3 y WaveOne Primary 25 0,08 (Dentsply Maillefer Ballaigues, Switzerland). Y en el G4 se instrumentó únicamente con WaveOne Primary 25 0,08. Los detritos extruidos durante la instrumentación, fueron recolectados en tubos Eppendorf que se pesaron previamente sin detritos, y luego se volvieron a pesar con los detritos para comparar la diferencia de valores. Tres pesos consecutivos se obtuvieron de cada tubo para registrar el peso promedio final de extrusión. En el análisis estadístico no hubo diferencia estadísticamente significativa entre los grupos 1 y 3, ni entre los grupos 2 y 4 ( $\mathrm{Sig}=1$ ). En el análisis de grupos homogéneos (G1-G3) y (G2-G4) existió diferencia estadísticamente significativa ( $P=0,019)$. En el G2 y G4 utilizado Reciproc y WaveOne como lo recomiendan las casas fabricantes ocasionan mayor extrusión de residuos comparado con los grupos G1 y G3 donde se hace un previamente un ensanchamiento del conducto radicular $(\mathrm{Sig}<0,05)$. Los resultados indican que todas las técnicas empleadas producen extrusión de residuos, sin embargo, la utilización de PathFile previo a la instrumentación reciprocante disminuye de manera significativa la extrusión hacia tejidos periapicales.

Palabras claves: Reciprocantes, glide path, extrusión de residuos

\section{Abstract}

The purpose of this in vitro study was to evaluate the amount $(\mathrm{mg})$ of dentinal debris extruded to periapex after rotary instrumentation with two types of reciprocating systems, with and without glide path prior. Forty mesial roots of mandibular molars were instrumented established working length (WL) in four groups (G), using G1. Pathfile 1 - 2 - 3 (Dentsply Maillefer) and Reciproc R25; 25 0,08 (VDW GmbH, Munich Germany) to WL, G2. RECIPROC R25 only to WL, G3. Pathfile 1-2 -3 and Waveone Primary 25 0,08 (Dentsply Maillefer Ballaigues, Switzerland) to WL, and G4. WaveOne Primary only to WL. The extruded debris during instrumentation were collected in preweighed Eppendorf tubes. Collectors Eppendorf tubes were weighed again with debris included. Three consecutive weights were obtained from each tube to the final weight of debris extrusion. There was no statistically significant difference between groups 1 and 3 , and between groups 2 and $4(P=1)$, the analysis of homogeneous subsets $G 1$ G3 and G2-G4, there are statistically significant $(P=0,019)$. Groups 2 and 4 using WaveOne and Reciproc and as recommended by the manufacturer produce more debris extrusion compared with Groups 1 and 3 that make previous glide path $(P>, 05)$. All instrumentation techniques were associated with extrusion; however, the use of Pathfile prior reciprocating instrumentation reduces significantly extrusion to periapical tissues.

Keywords: Reciprocating, glide path, debris extrusion 


\section{Introducción}

Uno de los factores que se ha estudiado, como una solución prometedora para evitar errores procedimentales durante la terapia endodóncica, es modificar el movimiento de trabajo de los instrumentos, evitando que sobrepasen el límite plástico de la aleación y así evitar la fractura, por eso se ha propuesto trabajar con limas en movimiento reciprocante, que tengan su movimiento cortante en sentido anti horario y un movimiento de liberación cuando regrese en sentido horario, de esta manera evitará que se atasque la punta en las paredes de conducto. Los instrumentos que trabajan bajo este principio son Reciproc de VDW y WaveOne de Dentsply Maillefer ${ }^{1,2,3}$.

Estos sistemas reciprocantes además proponen el uso de un único instrumento para la preparación del conducto radicular, lo que puede implicar ciertos riesgos tales como acumulación de estrés en la lima, ocasionar taponamientos en conductos muy estrechos por la conicidad de estos, cambios en la anatomía normal y pérdida de longitud de trabajo ocasionando sobre instrumentación, extrusión de residuos hacia el periápice y dolor posoperatorio. El hecho de que se maneje como instrumento único y éste haga el trabajo que tradicionalmente realizaban 4 o 5 instrumentos seriados lo hace más agresivo y la extrusión de detritos podría ser mayor ${ }^{4}$.

Una de las estrategias clínicas para conseguir un acceso en línea recta y mantener la anatomía radicular lo menos alterada posible es incluir en el protocolo de instrumentación un pre ensanchado coronal y de todo el conducto radicular, lo que no sólo mantiene la anatomía del conducto sino también se garantiza que los irrigantes lleguen al tercio apical evitando bloqueos con barrillo dentinario $\mathbf{5 , 6 , 7}$.

El desbridamiento completo del espacio del conducto radicular utilizando limas y soluciones irrigadoras es fundamental para conseguir el éxito de la terapia endodóncica. Durante la instrumentación pueden extruirse varias sustancias hacia el periápice, tales como irrigantes, restos de tejido pulpar, microorganismos y partículas de dentina resultando en inflamación postoperatoria. Este particular ha sido revisado en numerosos estudios. En uno de ellos, Al-Omari y Dummer, indican que existe menos extrusión cuando se utiliza el movimiento de fuerzas balanceadas y técnica corono - apical, mientras que con técnicas que involucran movimiento lineal ocurre más extrusión ${ }^{8}$.

La última generación de instrumentos NiTi Mwire, con movimiento reciprocante simula el movimiento lineal y de fuerzas balaceadas. Los avances en el diseño de los instrumentos tales como superficies radicales, profundidad de las estrías, diferentes conicidades y secciones transversales, así como los distintos movimientos que se emplean en su utilización podrían influir en la cantidad de detritos que se extruyen hacia el periápice ${ }^{8}$.

El objetivo de este estudio in vitro fue comparar la cantidad de detritos extruidos a través del foramen después de la preparación de conductos radiculares utilizando sistemas de lima única WaveOne (Dentsply Maillefer, Ballaigues, Suiza) y Reciproc VDW $\mathrm{GmbH}$, Munich, Alemania), con y sin la realización de un pre ensanchado radicular utilizando PathFile previo al empleo de ambos sistemas.

\section{Materiales y Métodos}

Se recolectaron 40 molares inferiores humanos descartados la posibilidad de ser restaurados ya sea por caries extensa, fracturas coronales o que hayan sido extraídos por problemas periodontales, el protocolo fue aprobado y revisado por el comité de Bioética de la Universidad San Francisco de Quito: Código: 2014-071T Versión No. 1 de mayo 27 de 2014 


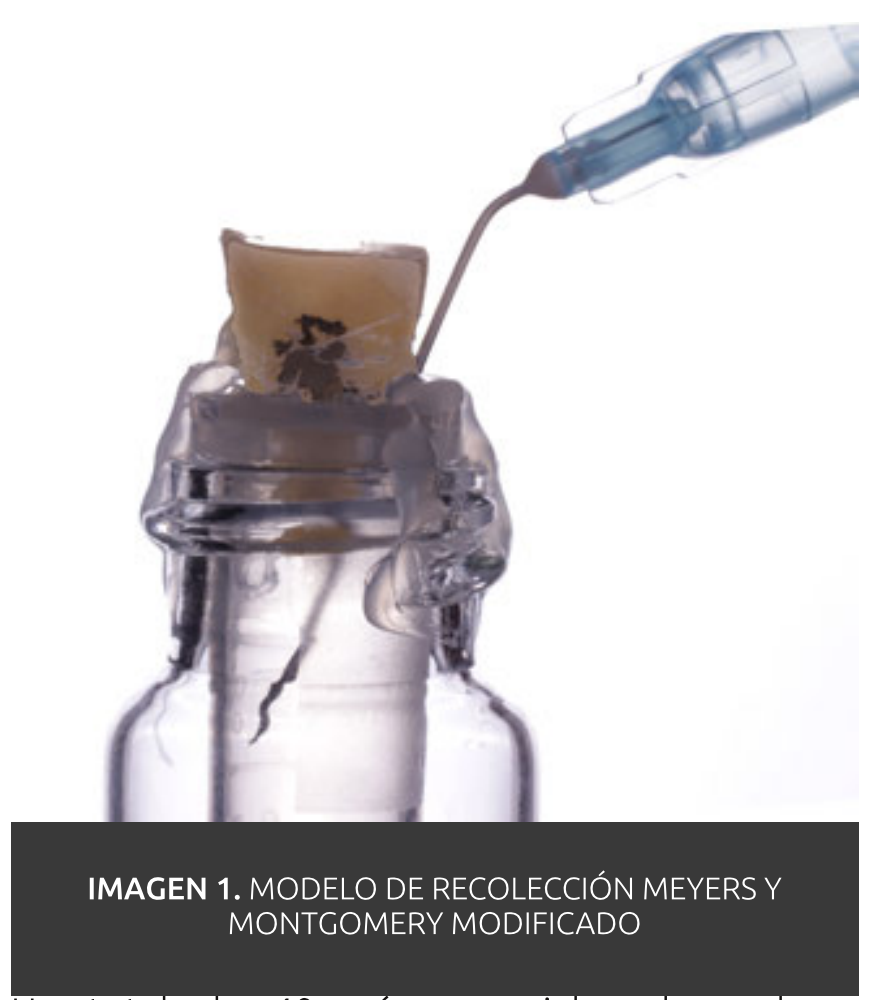

Un total de 40 raíces mesiales de molares mandibulares con ápices cerrados y anatomía Tipo II de Vertucci fueron seleccionadas para la investigación. Esto se verificó con una radiografía en sentido mesio - distal de las raíces seleccionadas. Los dientes se les dividió a través de la furca y las cúspides mesiales, con un explorador endodóncico DG16 se verificó las entradas a los conductos. En ambos conductos la longitud de trabajo se determinó empleando el Microscopio Operatorio a 16X utilizando una lima 10K, misma que al aparecer por el foramen, se disminuía $0,5 \mathrm{~mm}$ y esa fue considerada la longitud de instrumentación, la primera longitud obtenida sirvió como referencia para la realización de patencia

Para la recolección de los detritos se utilizó el modelo experimental de Myers y Montgomery ${ }^{9}$, los tapones de los tubos Eppendorf se eliminaron $y$ estos tubos fueron pesados en una balanza analítica de 10-5 g de precisión, tres pesos consecutivos se obtuvieron de cada recolector y se sacó un valor medio. En la apertura de cada tubo Eppendorf se colocó el diente a presión hasta la unión amelo cementaria; se colocó una aguja 27C para equilibrar las presiones interna y externa, como se indica en la imagen 1. El tubo
Eppendorf con el diente se colocó en un vial de vidrio para estabilizar la muestra, se selló con silicona caliente entre el vial y el Eppendorf, así como alrededor del diente para impermeabilizar el recolector.

Se dividió las muestras en 4 grupos $(n=10)$, la instrumentación se llevó a cabo por el mismo operador entrenado en todos los grupos, al igual que el pesaje de los tubos posterior a la preparación de las muestras.

- Grupo 1, se verificó la permeabilidad de los conductos con una lima 10K. A la longitud de trabajo se realizó el pre ensanchado mecanizado con Path File 1-2-3 (Dentsply Maillefer), se realizó patencia a un milímetro más de la longitud de trabajo con una lima 10 y luego se realizó la instrumentación final con Reciproc 25 0,08 (VDW).

- Grupo 2, se verificó la permeabilidad de los conductos con una 10K, a longitud de trabajo se instrumentó con Reciproc 25 0,08 .

- Grupo 3, se verificó la permeabilidad de los conductos con una lima $10 \mathrm{~K}$ a la longitud de trabajo. Luego se realizó el pre ensanchado mecanizado con Path File 1-2-3, se realizó patencia a un milímetro más de la longitud de trabajo con una lima 10 y luego se realizó la instrumentación final con WaveOne Primary 25 0,08.

- Grupo 4, se verificó la permeabilidad de los conductos con una lima 10K. A longitud de trabajo se instrumentó con WaveOne Primary $250,08$.

Todos los instrumentos se emplearon utilizando un motor VDW Silver Reciproc Motor, VDW y una pieza de reducción 6:1 Sirona, Bensheim, Germany. Los instrumentos PathFile a 300 rpm y 

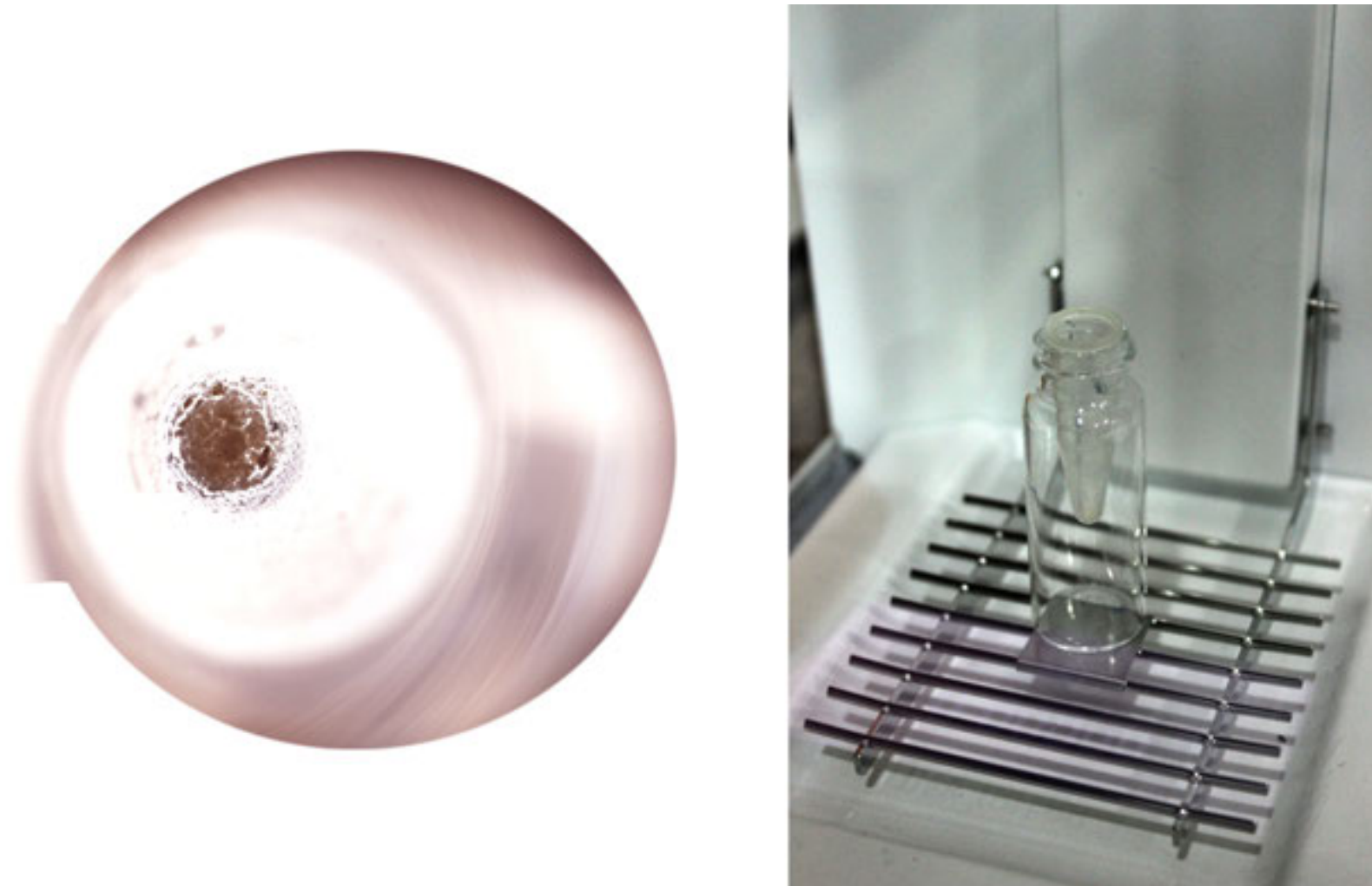

IMAGEN 2. PESO DE DETRITOS EN LA BALANZA ANALÍTICA

4.1 N de torque y los instrumentos Reciproc y WaveOne con los programas establecidos en el motor.

Después de cada instrumento, o después de los 3 picoteos con los instrumentos reciprocantes como recomiendan las casas fabricantes, se irrigó con agua destilada utilizando agujas Ultradent Navitip 29G/21 mm, la irrigación se realizó a $3 \mathrm{~mm}$ de la longitud establecida en las muestras.

Posterior a la instrumentación los dientes y la aguja se retiraron del tubo, los residuos adheridos a la raíz se lavaron con $1 \mathrm{~mL}$ de agua destilada y esto se recolectó en el tubo. Los tubos fueron almacenados a $105^{\circ} \mathrm{C}$ durante 12 horas en la estufa de calor seco para evaporar el agua destilada y mantener los residuos secos en el interior de los tubos.

Los tubos fueron nuevamente pesados en la balanza de $10^{-5} \mathrm{~g}$ de precisión, se obtuvieron de cada tubo tres pesos consecutivos para luego calcular la media. El valor de extrusión total se consiguió restado el peso de los tubos con detritos menos el peso de los tubos vacíos.

\section{Resultados}

Los principales resultados se indican en las tablas 1 y 2.

La media de extrusión de detritos más alta es la del grupo 4, donde únicamente se instrumentó con WaveOne Primary 25/0,08.

En términos generales el grupo 1 PathFile y Reciproc y en el grupo 3 PathFile y WaveOne son los que mejores resultados muestran por dejar menos residuos luego del procedimiento. El grupo1 PathFile y Reciproc es el que menor media de extrusión de detritos demuestra, pero entre estos dos grupos la diferencia relativa o el resultado diferencial no es significativo.

Estos resultados también nos indica que los grupos donde se realizó el pre ensanchado radicular con PathFiles 1-3 extruyeron menos detritos que los grupos donde no se realizó este 


\begin{tabular}{|c|c|c|c|c|}
\hline & Media & Desviación típica & Mínimo & Máximo \\
\hline PathFile y Reciproc & 0,72 & 0,07 & 0,63 & 0,83 \\
\hline Reciproc & 1,07 & 0,31 & 0,62 & 1,61 \\
\hline PathFile y WaveOne & 0,73 & 0,22 & 0,48 & 1,06 \\
\hline WaveOne & 1,08 & 0,33 & 0,53 & 1,45 \\
\hline Total & 0,90 & 0,30 & 0,48 & 1,61 \\
\hline
\end{tabular}

TABLA 1. ESTADÍSTICA DESCRIPTIVA DE LOS DATOS. MEDIA EN MILIGRAMOS DE DETRITOS EXTRUIDOS APICALMENTE UTILIZANDO DIFERENTES TÉCNICAS DE INSTRUMENTACIÓN

pre ensanchado, existiendo diferencias estadísticamente significativas entre dichos grupos.

\section{Discusión}

La preparación biomecánica con instrumentos manuales y mecanizados, sumados a la irrigación del conducto radicular, puede desencadenar una reacción inflamatoria al forzar la salida del contenido intraconducto como partículas de dentina, tejido pulpar necrótico o microorganismos hacia la región periapical causando dolor postoperatorio al hospedador 10,11 .

Para hacer una valoración objetiva, en el presente estudio se utilizaron las limas rotatorias Reciproc 25 0,08 y WaveOne Primary File 25 0,08, las cuales presentan el mismo tamaño de la punta y la conicidad inicial; de esta manera poder tener los mismos límites de comparación en cuanto a la instrumentación.

Reciproc presentó una media de extrusión ligeramente menor a la de WaveOne con o sin el pre ensanchamiento con path si. Al comparan entre los grupos 1 y 3 con los grupos 2 y 4 en términos de extrusión, se puede ver que el peso de detritos extruidos es menor cuando se realiza un pre ensanchado.
Pasqualini en el 2012 determinó que el dolor post operatorio cuando se utiliza PathFiles es menor y la resolución de la sintomatología es más rápida, lo que corrobora los resultados de esta investigación, ya que cuando hay menos extrusión el dolor post operatorio es menor al igual que el riesgo de agudizaciones ${ }^{\mathbf{1 2}}$.

Myers y Montgomery sugirieron una reevaluación del tapón de la dentina apical debido a los beneficios potenciales de reducir la cantidad de desechos apicales extruidos e irrigantes y la prevención de sobreinstrumentación en combinación con la extrusión de materiales de relleno ${ }^{9}$. En el estudio de Lambrianidis demuestra que realizar patencia apical se asocia con menos desechos extruidos apicalmente en comparación con los dientes en la que la constricción se mantuvo intacta ${ }^{13}$. Un dato importante al utilizar la lima de pasaje es que asegura la llegada de soluciones irrigadoras al tercio apical, situación clínica que Vera y cols. encontraron en un estudio in vivo ${ }^{13}$. A estos datos de patencia apical podemos decir que el uso de lima de pasaje sumado a un pre ensanchamiento mecanizado facilita la instrumentación con sistemas reciprocantes, esto clínicamente se puede comprobar por la facilidad con la que llega el instrumento a la longitud de trabajo establecida, si previamente se utiliza instrumentos PathFile y se hace patencia junto con irrigación entre cada instrumento. 


\begin{tabular}{|c|c|c|c|c|c|}
\hline \multicolumn{6}{|c|}{ HSD de Tukey } \\
\hline \multirow[t]{2}{*}{ (I) Grupo } & \multirow[t]{2}{*}{ (J) Grupo } & \multirow{2}{*}{$\begin{array}{l}\text { Diferencia de } \\
\text { medias } \\
(\mathrm{I}-\mathrm{J})\end{array}$} & \multirow[t]{2}{*}{ Sig. } & \multicolumn{2}{|c|}{$\begin{array}{l}\text { Intervalo de confianza al } \\
95 \%\end{array}$} \\
\hline & & & & $\begin{array}{l}\text { Límite } \\
\text { inferior }\end{array}$ & $\begin{array}{l}\text { Límite } \\
\text { superior }\end{array}$ \\
\hline \multirow{3}{*}{$\begin{array}{l}\text { Path File y } \\
\text { Reciproc }\end{array}$} & Reciproc &,$- 34567 *$ & 0,021 & $-0,65$ & $-0,0413$ \\
\hline & $\begin{array}{l}\text { Path File y } \\
\text { WaveOne }\end{array}$ & $-0,00134$ & 1 & $-0,3057$ & 0,303 \\
\hline & WaveOne &,$- 35134 *$ & 0,018 & $-0,6557$ & $-0,047$ \\
\hline \multirow[t]{3}{*}{ Reciproc } & $\begin{array}{l}\text { Path File y } \\
\text { Reciproc }\end{array}$ & ,34567* & 0,021 & 0,0413 & 0,65 \\
\hline & $\begin{array}{l}\text { Path File y } \\
\text { WaveOne }\end{array}$ & ,34434* & 0,021 & 0,04 & 0,6487 \\
\hline & WaveOne & $-0,00567$ & 1 & $-0,31$ & 0,2987 \\
\hline \multirow{3}{*}{$\begin{array}{l}\text { Path File y } \\
\text { WaveOne }\end{array}$} & $\begin{array}{l}\text { Path File y } \\
\text { Reciproc }\end{array}$ & 0,00134 & 1 & $-0,303$ & 0,3057 \\
\hline & Reciproc &,$- 34434^{*}$ & 0,021 & $-0,6487$ & $-0,04$ \\
\hline & WaveOne &,$- 35000 *$ & 0,019 & $-0,6543$ & $-0,0457$ \\
\hline \multirow[t]{3}{*}{ WaveOne } & $\begin{array}{l}\text { Path File y } \\
\text { Reciproc }\end{array}$ & ,35134* & 0,018 & 0,047 & 0,6557 \\
\hline & Reciproc & 0,00567 & 1 & $-0,2987$ & 0,31 \\
\hline & $\begin{array}{l}\text { Path File y } \\
\text { WaveOne }\end{array}$ & ,35000* & 0,019 & 0,0457 & 0,6543 \\
\hline
\end{tabular}

\section{TABLA 2. COMPARACIONES MÚLTIPLES DE GRUPOS UTILIZANDO LA PRUEBA DE TUKEY}

Casi todos los estudios sobre la extrusión han utilizado los dientes de una sola raíz con conductos relativamente rectos de $<5-10$ grados de curvatura. Leonardi y col. en el 2007 se enfocaron en las raíces con mayores curvaturas y determinaron que el grado de la curvatura no fue determinante en el peso de material extruido. En esta investigación se escogió las raíces mesiales de molares inferiores ya que la anatomía curva de estos conductos se logra mantener en la mayoría de casos con Reciproc 25 o WaveOne Primary ${ }^{\mathbf{1 4}}$.

Resultados similares a los de este estudio se encontraron en investigaciones como la de Kocak en el 2013 quien comparó los sistemas: SAF, Reciproc, ProTaper y Revo-S. Sus resultados indican que no existe diferencia significativa en el peso de extrusión, sin embargo, el que menos extruye es la lima Reciproc y el que más extruye es ProTaper ${ }^{15}$.

El estudio de Bürklein y cols se asegura que independientemente de la técnica que se utilice en un tratamiento de conductos existirá extrusión. También se indica que cuando se utiliza sistemas de secuencia completa con movimiento de rotación continua existirá menos extrusión que si se utiliza sistemas de lima única con movimiento reciprocante ${ }^{16}$.

El dato de extrusión de WaveOne comparado con Recirproc en el estudio Bürklein es que R40 extruye más detritos que WaveOne large, este 
resultado se da porque los instrumentos que se comparan tienen conicidades distintas WaveOne large es 40 0,08 mientras que Reciproc es 40 0,06 16.

Los resultados un vitro no son $100 \%$ extrapolables a la clínica, entre otras cosas debido a la ausencia de una presión de retorno física proporcionada por los tejidos periapicales que limita la extrusión de residuos ${ }^{\boldsymbol{9}}$. Sin embargo, nuestros resultados nos dan una buena guía para mejorar las técnicas de instrumentación rotatoria.

Después del trabajo de simulación clínica, análisis estadístico y resultados, se valida la Hipótesis planteada en esta tesis, ya que comprobamos que la realización previa de ensanchamiento del conducto es un determinante en la cantidad de residuos que se extruyen hacia la región periapical, siendo menor la extrusión en los grupos que se trabaja con PathFile que en los que se trabaja como recomienda la casa fabricante de los sistemas reciprocantes de lima única.

\section{Conclusiones}

- La realización de un pre ensanchamiento del canal radicular con limas PathFile combinado con técnicas de instrumentación reciprocante, demuestra disminuir de manera significativa la cantidad de detritos extruidos hacia los tejidos periapicales.

- No hubo diferencia estadísticamente significativa en los valores de extrusión periapical de detritos entre la instrumentación rotatoria reciprocante de WaveOne 25 0,008 y Reciproc 25 0,08.

\section{Bibliografía}

1. Yared G. Canal preparation using only one $\mathrm{Ni}$-Ti rotary instrument: preliminary observations. International Endodontic Journal. 2008;41(4):339-44.
2. Bürklein $S$, Hinschitza $K$, Dammaschke $T$, Schäfer E. Shaping ability and cleaning effectiveness of two single-file systems in severely curved root canals of extracted teeth: Reciproc and WaveOne versus Mtwo and ProTaper. International Endodontic Journal. 2012; 45(5):449-61.

3. De-Deus G, Moreira E, Lopes H, Elias C. Extended cyclic fatigue life of F2 ProTaper instruments used in reciprocating movement. International Endodontic Journal. 2010;43: 1063-1068.

4. You S. Bae K, Baek S, Kum K, Shon W, Lee W. Lifespan of One Nickel-Titanium Rotary File with Reciprocating Motion in Curved Root Canals. J Endod. 2010;36(12):1991-1994.

5. Berutti E. Use of Nickel-Titanium Rotary PathFile to Create the Glide Path: Comparison with Manual Preflaring in Simulated Root Canals. JOE. 2009;35(3):408-4012

6. Pasqualini $D$, Mollo $L$, Scotti $N$, Cantatore G, Castellucci A, Migliaretti G, Berutti E. Postoperative Pain after Manual and Mechanical Glide Path: A Randomized Clinical Trial. JOE. 2012; 38(1):32-36.

7. Pasqualini D, Bianchi C, Paolino DS, Mancini L, Cemenasco A, Cantatore G, Castellucci A, Berutti E. Computed Micro-Tomographic Evaluation of Glide Path with NickelTitanium Rotary PathFile in Maxillary First Molars Curved Canals. JOE. 2012;38(3):389-393.

8. Tanalp J, Güngör T. Apical extrusion of debris: a literature review of an inherent occurrence during root canal treatment. International Endodontic Journal. 2014:47(3):211-221. 
9. Myers GL, Montgomery S. A comparison of weights of debris extruded apically by conventional filing and Canal Master techniques. J Endod.1991;(6):17:275-279.

10. Ruiz-Hubard E, Gutmann J, Wagner M. A Quantitative Assessment of Canal Debris Forced Periapically during Root Canal Instrumentation Using Two Different Techniques. JOE. 1987;13(12):554-558.

11. Hülsmann $M$, Hahn W. Complications during root canal irrigation. Int Endod J. 2000;33(3):186-93.

12. Pasqualini $D$, Mollo $L$, Scotti $N$, Cantatore G, Castellucci A, Migliaretti G, Berutti E. Postoperative Pain after Manual and Mechanical Glide Path: A Randomized Clinical Trial. J Endod. 2012;38(1):32-36.

13. Vera J, Arias A, Romero M. Effect of Maintaining Apical Patency on Irrigant Penetration into the Apical Third of Root Canals When Using Passive Ultrasonic Irrigation: An In Vivo Study. J Endod. 2011 Sep;37(9):1276-8.
14. Leonardi L., Atlas D., Raiden G. Apical extrusion of debris by manual and mechanical instrumentation. Braz Dent J. 2007;18(1):16-19.

15. Kocak S, Koçak $M$, Sağlam B, Türker $S$, Sağsen B, Er Ö. Apical Extrusion of Debris Using Self-Adjusting File, Reciprocating Single-file, and 2 Rotary Instrumentation Systems. J Endod. 2013;39(10):1278-1280.

16. Bürklein S. Apically Extruded Debris with Reciprocating Single-File and Fullsequence Rotary Instrumentation Systems. J Endod 2012;38(6):850-852. 